\title{
Análise de uma Arquitetura da Informação associada a Multimodalidade na capacitação de profissionais de alto desempenho
}

\author{
Analysis of an Information Architecture associated with Multimodality in the training of high \\ performance professionals
}

Tomás Roberto Cotta Orlandi

Doutorando em Ciências da Informação na Universidade de Brasília - UnB.

Professor de graduação nas áreas de análise de sistemas e modelagem de dados. Professor de pós-graduação nas áreas de Gerenciamento de Projetos, BSC voltado para TI e Engenharia de Software.

E-mail: tomasroberto@gmail.com

Claudio Gottschalg Duque

Doutor em Linguística Computacional no Angewandte Sprachwissenschaft und Computerlinguist - JustusLiebig-Universität Giessen e em Produção e Gestão da Informação no Programa de Pós-Graduação em Ciência da Informação pela Escola de Ciência da Informação. Professor Adjunto da Faculdade de Ciência da Informação da Universidade de Brasília e do Programa de PósGraduação em Ciência da Informação - UnB. E-mail: klaussherzog@gmail.com

\section{Resumo}

A capacitação permanente de profissionais, em qualquer área do conhecimento, é um desafio a ser alcançado nos dias de hoje. Com profissionais de alto desempenho não é diferente, eles devem dominar uma ampla gama de disciplinas que vão desde relacionamentos humanos ao domínio da gestão de riscos empresariais. A pesquisa apresenta uma análise da aplicação prática dos conceitos de arquitetura da informação, espaços informacionais e multimodalidade como proposta de atendimento a essas demandas de capacitação desses profissionais. A metodologia empregada foi da pesquisa aplicada, utilizando questionários direcionados aos profissionais de alto desempenho, para levantamento dos dados. Os resultados da pesquisa demonstram uma boa aceitação do modelo, inicialmente com $94 \%$ das avaliações concentradas entre as notas de 8 a 10, conceitos de bom a ótimo para as perguntas formuladas. A pesquisa indica que a multimodalidade aplicada na capacitação profissional pode auxiliar, significativamente, o alcance das necessidades de informação do educando, para que ele possa desempenhar suas atividades técnicas e gerenciais cotidianas.

Palavras-Chave: Arquitetura da Informação. Espaços Informacionais. Multimodalidade.

\begin{abstract}
The permanent training of professionals in any area of knowledge is a challenge to be achieved today. With highperforming professionals is no different, they must master a wide range of disciplines ranging from human relationships to the domain of enterprise risk management. The research presents an analysis of the practical application of the concepts of information architecture, informational spaces and multimodality as a proposal to meet these demands of training of these professionals. The applied methodology was applied research, using questionnaires directed to professionals of high performance, to collect the data. The results of the research demonstrate a good acceptance of the model, initially with $94 \%$ of the evaluations concentrated between grades 8 to 10 , concepts from good to great for the questions asked. The research indicates that the multimodality applied in the professional training can significantly help the reach of the information needs of the student, so that he can carry out his daily technical and managerial activities.
\end{abstract}

Keywords: Information Architecture. Informational Spaces. Multimodality. 


\section{Introdução}

A Arquitetura da Informação é uma metodologia de desenho (MACEDO, 2005), que se aplica a qualquer ambiente informacional, sendo este compreendido como um espaço localizado em um contexto, constituído por conteúdos em fluxo, que serve a uma comunidade de usuários. A finalidade da Arquitetura da Informação é viabilizar o fluxo efetivo de informações por meio do desenho de ambientes informacionais.

Para Oliveira (2012) a Ciência da Informação trata de informação e também das questões relativas à sua estruturação. Nessa ciência, são encontradas as ferramentas necessárias para o estabelecimento dos chamados "Espaços Informacionais" que podem ser compostos por grandes quantidades de informação, mas com elevado grau de descentralização e fragmentação. Pode se entender espaço informacional como um recorte de uma teia de informações organizacionais. Para alcançar sua finalidade de concepção, o espaço informacional precisa ter a capacidade de suprir as necessidades especificas de informações de indivíduos e também de suas organizações. O espaço informacional deve gerar relações eficientes entre os indivíduos e as informações, de maneira a proporcionar ao indivíduo a capacitação necessária para que seja possível atingir suas metas e objetivos.

Kress e Van Leeuwen (2001) definem Multimodalidade como o uso de vários modos semióticos no projeto de um produto ou evento semiótico, ou: "É a terminologia comum para todos os dados semióticos". O conceito de multimodalidade brota da Teoria da Semiótica, mais especificamente, da Semiótica Social. Para Barros (2005), a semiótica prima pelo estudo do texto, mais especificamente, focando em explicar "o que o texto diz e como ele faz para dizer o que diz" (BARROS, 2005). Em outras palavras, a semiótica prima não só pelo estudo daquilo que é dito pelo texto, como também pelas estratégias textual-discursivas traçadas pelo autor do texto, a fim de exteriorizar o seu dizer. Diante disto, este campo de estudo se debruça sobre as mais distintas construções linguísticas do texto, para materializar seu dizer.

Os Espaços Informacionais devem ser projetados de forma estruturada para atender objetivos específicos de adequação das necessidades dos usuários, delimitando apenas as informações indispensáveis e relevantes de uma grande quantidade de informações.

A capacitação permanente de profissionais, em qualquer área do conhecimento, é um desafio a ser alcançado nos dias de hoje. Com profissionais de alto desempenho não é diferente. Estes profissionais devem dominar uma ampla gama de disciplinas que vão desde 
relacionamentos humanos ao domínio da gestão de riscos empresariais. Para esta finalidade, vislumbramos a possibilidade de utilização de espaços informacionais multimodais, com textos, sons, imagens e vídeos, que podem proporcionar um conjunto de informações estruturadas voltadas para uma capacitação profissional.

Apesar da grande profusão de portais e sítios voltados para capacitações, não existe um modelo consagrado de Arquitetura da Informação associada a Multimodalidade que, aplicado a espaços informacionais, atenda às necessidades de informação de profissionais de alto desempenho. Desta maneira, este trabalho pretende abordar o seguinte problema de pesquisa:

Como uma Arquitetura da Informação associada a Multimodalidade pode auxiliar na capacitação de profissionais de alto desempenho?

O presente artigo relata uma pesquisa aplicada de maio a julho de 2017, em que foi construído um espaço informacional multimodal com o objetivo de capacitar profissionais de alto desempenho de uma grande organização pública nacional. Esse relato está dividido nas seções a seguir, apresentando primeiramente o referencial teórico, o objeto da pesquisa, a metodologia utilizada, os resultados e as considerações finais.

\section{Conceitos da Pesquisa}

Em Lyra (2012) é citado que uma forma de projetar os Espaços Informacionais é a Arquitetura da Informação - AI, uma das linhas de pesquisa da Ciência da Informação. A AI vem se consolidando e ganhando espaço nas últimas décadas dentro da Sociedade da Informação, considerando uma intensa e significativa evolução em seus conceitos e definições.

Lyra (2012) sugere que a Arquitetura da Informação se coloca a serviço das empresas ocupando-se com a identificação das necessidades de informações, com a compreensão dos conteúdos e dos desafios de organizá-las de maneira a torná-las úteis com a prontidão necessária. Quando bem definida e estabelecida em comum acordo com todos os usuários da informação, essa arquitetura auxilia que todas as partes envolvidas na tomada de decisão utilizem a informação e falem a mesma língua.

O produto final de uma Arquitetura da Informação é uma estrutura que utiliza as tecnologias disponíveis para dar forma e controlar o ambiente de modo que um grupo especificado de atividades humanas possa ser empreendido com maior eficiência (Lyra, 2012). 
González de Gómez (1990) salienta que o objeto de estudo da Ciência da Informação é o sistema formal intermediário de recuperação da informação e para Saracevic (1995) a natural interdisciplinaridade da CI se apresenta na relação com a ciência da computação, que trata de algoritmos relacionados à informação.

Já para Buckland (1991) a referência de informação como coisa, por ser um objeto informativo é fundamental, trata da informação trabalhada, numa abordagem de coisas, no sentido que podem nos informar sobre algo. Para ele o conceito de documento é qualquer recurso informacional físico, trabalhando sobre a perspectiva de modelos designados para representar ideias e objetos como a exemplo de: obras de artes, livros, textos, imagens, áudios e vídeos.

Dionisio $(2005 ; 2011)$ destaca que as discussões concernentes à multimodalidade se propagaram consideravelmente. Vários pesquisadores de diversos campos de estudo: Análise do Discurso, Análise Crítica do Discurso, Análise do Discurso de Linha Francesa, Linguística Aplicada, Linguística de Texto, Pedagogia, Psicologia, Semiótica, Semiótica Social, Sociologia, tem estudado esta temática, abordando as distintas maneiras de como este conceito se materializa nas múltiplas formas da linguagem, seja escrita, oral e visual.

As tecnologias não se resumem a máquinas apenas - ganharam um novo papel, humanizado, com as preocupações da interação homem-máquina e a socialização do conhecimento (PINHEIRO, 2013). Em Capurro (2007) a Ciência da Informação nasce com um paradigma físico, depois questionado por um enfoque cognitivo idealista e individualista, sendo este por sua vez substituído por um paradigma pragmático e social. Já para Hjorland (2002), a visão sócia cognitiva tende a dar tratamento epistemológico a assuntos da psicologia no conhecimento do indivíduo numa perspectiva histórica, cultural e social.

Para Luna (2002) o texto é concebido como algo resultante da atuação das múltiplas formas da linguagem, o texto não é construído linguisticamente apenas, por meio da escrita. Ele pode se materializar através da linguagem escrita, oral e imagética, bem como da articulação e integração destas modalidades. Para Xavier (2006) o texto é uma prática comunicativa, materializada por intermédio das múltiplas modalidades da linguagem, tais como: verbal (escrita e oral) e não-verbal (visual).

A competência é um conjunto de conhecimentos, habilidades e atitudes necessárias à consecução de determinado propósito (DURAND, 1998). Conhecida pela sigla CHA (Conhecimento, Habilidade e Atitude) competência é necessária à realização de algo, ou seja, é 
aplicada. A competência a ser estudada é a do gerente do projeto, composta por conhecimentos, habilidades e atitudes e necessárias à condução de um projeto, representando a consecução de determinado propósito.

Competências têm sido estudadas em três níveis: individual, grupal (ou força de trabalho) e corporativo. O conceito de competências essenciais (core competencies) desenvolvido por Prahalad e Hamel (1990) relaciona-se ao nível corporativo. Existem padrões, como, por exemplo, o People CMM - Capability Maturity Model (SEI, 2001), que estão focados na força de trabalho de uma determinada organização ou pertencente a algum grupo profissional. Todos esses padrões agrupam práticas de desenvolvimento de competências.

Para Mazurkiewicz (2010) um profissional de alto desempenho possui competências especificamente requeridas ou desenvolvidas para o negócio da empresa:

Desde o seu início, a IBM tem se concentrado no desenvolvimento dos melhores profissionais de negócios e de tecnologia do mundo. Com isso, a companhia conquistou uma vantagem competitiva ao criar e cultivar um tipo singular de pensador e trabalhador: o "IBMista". Durante os últimos 15 anos, essa dedicação significou pesquisar características específicas demonstradas pelo comportamento dos melhores IBMistas - características que chamam de competências. Essas competências estão sistematicamente integradas em um amplo conjunto de processos de desenvolvimento, pois reconhecem que é necessário evoluir à medida que as necessidades de negócios e o mundo se transformam. Ao refletir essa mudança significativa em nos negócios atuais, a IBM identificou e validou um novo conjunto de nove características - as IBM Competencies - que descrevem e definem expectativas do IBMista moderno - como ele ou ela precisa se comportar para garantir que a IBM está em sua melhor forma. Quando um IBMista está em sua melhor forma, ele:

1. Enfrenta desafios (Embrace challenge)

2. É parceiro do sucesso dos clientes (Partner for clients' success)

3. Colabora globalmente (Collaborate globally)

4. Atua em uma perspectiva sistêmica (Act in a systemic perspective)

5. Constrói confiança mútua (Build mutual trust)

6. Influencia através de expertise (Influence through expertise)

7. Trabalha para a contínua transformação (Continuously transform)

8. Comunica para ter impacto (Communicate for impact)

9. Ajuda IBMistas a ter sucesso (Help IBMers succeed)

Essas competências atualizadas refletem a forma real como os negócios e o mundo estão mudando. Essa nova abordagem de liderança eleva e une todos os programas existentes de desenvolvimento de carreira da IBM, oferecendo a IBMistas do mundo inteiro um quadro mais claro do conhecimento, capacitação e comportamentos gerais de liderança necessários para ter sucesso no mercado de trabalho de hoje (MAZURKIEWICZ, 2010, p. 6).

Observa-se que o denominado profissional de alto desempenho tem um perfil de atuação destacado, uma vez que assume desafios e busca sempre colaborar e compartilhar conhecimentos adquiridos, comunicando e influenciando outros profissionais para obtenção de resultados para as organizações, clientes e outros profissionais. 
Losada (1999) propõe que equipes de alto desempenho podem ser medidas por três variáveis na visão dos clientes: a rentabilidade, a satisfação dos clientes e a avaliação $360^{\circ}$ que são usualmente feitas nas empresas. O autor então criou um índice baseado nestas três variáveis, ou seja, se a equipe era rentável, mantendo os clientes satisfeitos e demonstraram uma avaliação elevada, era então, uma equipe de alto desempenho.

Para Vargas (2014) um alto nível de colaboração e inovação é observado em equipes de alto desempenho que auxiliam o time a produzir resultados superiores de forma consistente. Ainda na visão deste autor podem se caracterizar ações e comportamentos nesses times, elencados como nove deveres da equipe de alto desempenho:

1. - Metas e planos claros;

2. - Comunicação efetiva;

3. - Relações positivas entre os membros;

4. - Papéis e responsabilidade claros;

5. - Confiança mútua;

6. - Eficaz tomada de decisões;

7. - Valorização e promoção da diversidade;

8. - Gerenciamento bem-sucedido de conflitos;

9. - Reconhecimento do trabalho e de oportunidades de desenvolvimento.

\section{Modelos pesquisados}

Buscando atender às necessidades de informação de profissionais de alto desempenho, foram levantados modelos que poderiam possibilitar a implementação de um espaço informacional multimodal. Os levantamentos apontaram para dois possíveis modelos de arquitetura da informação, ambos desenvolvidos por pesquisadores brasileiros, como teses de doutoramento dos seus respectivos programas de pós-graduação. 
3.1.O Modelo da Metodologia de Desenvolvimento de Ambientes Informacionais Digitais

Camargo (2010) apresenta um modelo para desenvolvimento de ambientes informacionais digitais, que pode ser considerado como um conjunto de passos além do processo de desenvolvimento de software tradicional, pois sugere novos processos e atividades para os profissionais da informação. Esse modelo possui enfoque no tratamento dos objetos de conteúdo do ambiente digital, de acordo com necessidades dos usuários. Essa metodologia de desenvolvimento proposta por Camargo (2010, p. 153) considera:

1. O tratamento funcional do ambiente, que envolve a especificação das funcionalidades e dos serviços do ambiente de acordo com as necessidades dos usuários;

2. O tratamento estrutural do ambiente, que envolve a estruturação do ambiente, abordando as opções estruturais da arquitetura e os fluxos informacionais:

3. O tratamento do conteúdo, que envolve a representação e descrição da informação por meio da análise semântica, sintática e pragmática, bem como por meio dos processos de classificação, catalogação e indexação, considerando o contexto do usuário e uso da informação pelo mesmo:

4. O tratamento navegacional do ambiente, que envolve a navegação do conteúdo, considerando o comportamento e modo de interação do usuário e do ambiente;

5. O tratamento da aparência visual do objeto de conteúdo, que envolve a apresentação da informação por meio da rotulagem e da formatação e editoração do conteúdo, considerando a usabilidade e acessibilidade. 


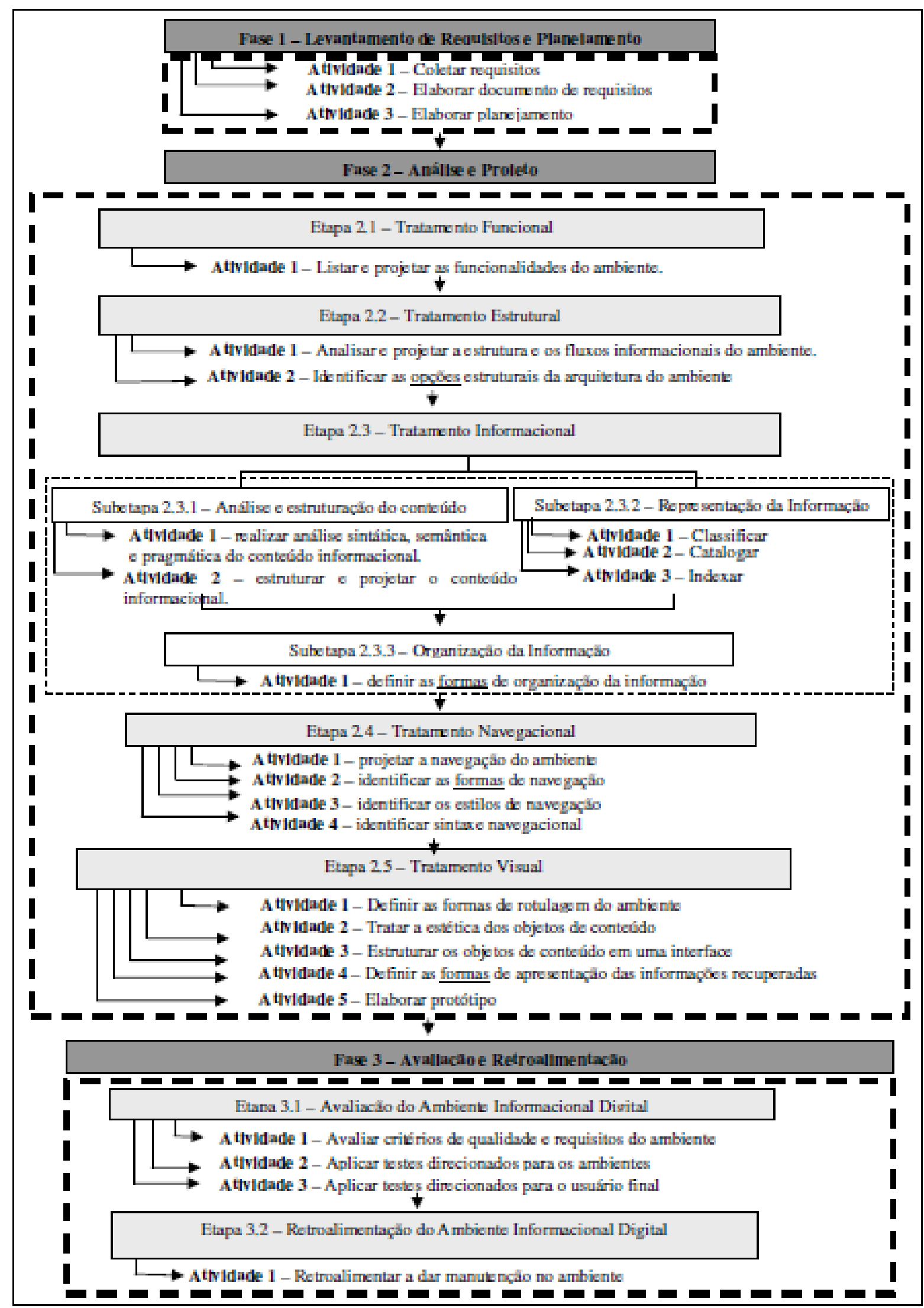

Figura 1: Metodologia de Desenvolvimento de Ambientes Informacionais Digitais Fonte: Camargo (2010, p. 154) 


\subsection{O Modelo DAIM:CSCL}

Em Steinmetz et al. (2016) identificamos um conjunto inicial de características básicas que devem ser contempladas em um ambiente CSCL, da sigla em inglês Computer Supported Collaborative Learning (traduzido pelos autores como Ambientes Informacionais Colaborativos de Apoio ao Processo de Ensino-Aprendizagem), segundo os preceitos da uma AI associada a Multimodalidade e da teoria da relevância:

1. O ambiente deve ser concebido/adaptado ao contexto dos estudantes/usuários;

2. O ambiente deve oferecer vários modos comunicacionais, conforme prevê a definição do termo multimodalidade;

3. O ambiente deve considerar a usabilidade e a ergonomia a fim de influenciar positivamente a interação dos usuários;

4. O ambiente deve apresentar uma arquitetura da informação que permita a inclusão, organização e recuperação das informações de forma simples e eficiente;

5. Em complemento aos itens 3 e 4 o ambiente deve oferecer um design centrado no humano. Ainda em Steinmetz et al. (2016) os autores consideraram o Modelo de Ciclo de Vida do Desenvolvimento de Sistemas proposto por Gordon e Gordon (2006), para propor um diagrama (figura 2), apresentando um conjunto de fases a serem executadas para acomodar características básicas de uma AI associada a Multimodalidade para ambientes CSCL.

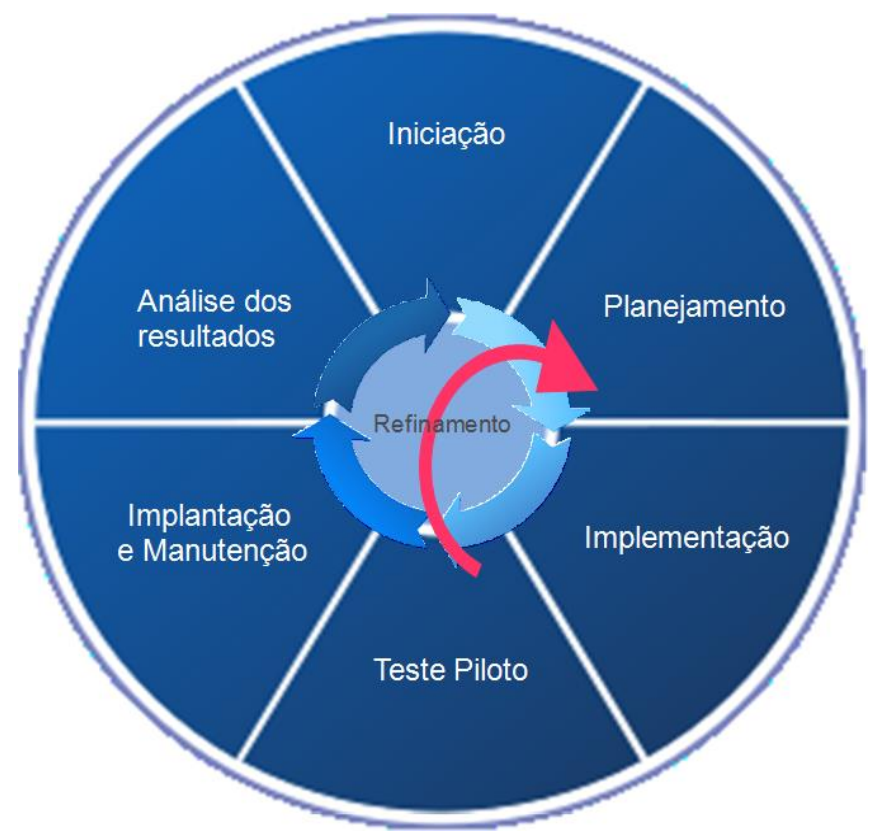

Figura 2: Diagrama de fases para concepção de uma Arquitetura da Informação Multimodal para ambientes $C S C L-$ DAIM:CSCL.

Fonte: Anais - X Encontro de Docentes de Escolas de Biblioteconomia e Ciência da Informação do MERCOSUL EBCIM 2016 (STEINMETZ et al., 2016) 
Na figura 2, que apresenta o DAIM:CSCL, temos as seguintes fases:

- Iniciação: ocorre a identificação da demanda, identificação do público alvo, identificação do contexto do público alvo, definição da equipe responsável pelo processo, levantamento inicial das necessidades, definição dos objetivos gerais a serem alcançados.

- Planejamento: construção das especificações detalhadas para que os objetivos definidos na iniciação sejam alcançados, por meio do projeto das interfaces que deve considerar recursos multimodais pertinentes ao contexto dos usuários; o projeto de banco de dados; o projeto transacional que deve comportar as solicitações postadas na interface retornando os resultados do banco de dados; e as características físicas do sistema, sendo o conjunto das interfaces, banco de dados e transações os elementos que definem a Arquitetura da Informação Multimodal. Ainda, podemos considerar a possibilidade de aquisição de hardware e softwares necessários para viabilizar a execução do processo. Finalmente, devem ser preparados os testes do sistema.

- Implementação: é desenvolvimento do ambiente segundo as especificações definidas na fase de planejamento. Aqui é feita a codificação ou aquisição do software, a aquisição do hardware e o teste do novo sistema.

- Teste Piloto: aprimora o novo ambiente considerando que o trabalho já foi conceituado e planejado nas fases anteriores. Neste momento o ambiente $C S C L$ deve ser alimentado com as informações referentes ao curso a ser ministrado, sendo importante que estes conteúdos estejam adequados ao contexto dos usuários e aos modos comunicativos definidos nas etapas de iniciação e planejamento.

- Implantação e Manutenção: utilização do ambiente em larga escala, sendo efetuadas as manutenções corretivas e adaptativas pertinentes, bem como do registro histórico destas manutenções, dos índices de satisfação dos usuários e sugestões de melhorias.

- Análise dos Resultados: completa o ciclo de aprimoramento do processo buscando revisar os relatórios do que foi executado, verificando se o esforço alcançou os objetivos pretendidos inicialmente. Pode ser vista também como uma fase de aprendizado para a equipe que executa o processo, sendo importante que os registros sejam mantidos através de todo o ciclo do processo. 


\section{Metodologia da Pesquisa} profissionais de alto desempenho

Esse estudo adota uma abordagem quantitativa na coleta e uma abordagem qualitativa na análise dos dados que, conforme Richardson (2008), deste modo tem-se a precisão e profundidade necessária para o estudo. Também conforme preconizado por Denzin e Lincoln (2006), as questões sociais são analisadas de forma melhor por um viés qualitativo, sendo os dados de cunho quantitativo.

O presente estudo é classificado como pesquisa aplicada, pois objetiva gerar conhecimento para aplicação prática dirigida e solução de problemas específicos. A área específica do estudo é a Arquitetura da Informação. Envolveu levantamento bibliográfico, entrevistas com pessoas que tiveram experiências práticas com o problema pesquisado. As entrevistas nesta pesquisa foram realizadas sob a forma de questionário.

Essa pesquisa foi realizada entre 01/05/2017 e 17/07/2017, quando foram coletados os últimos dados das respostas dos questionários, respeitando o prazo final para o preenchimento das respostas a 20 perguntas, sendo 05 perguntas específicas sobre os objetos multimodais, (vídeos, áudios, textos, etc.) aplicados como recurso didático para capacitação dos profissionais de alto desempenho.

\subsection{Ambiente da Pesquisa}

A pesquisa foi aplicada em uma empresa pública brasileira de grande porte, com presença nacional, que tem como preocupação capacitar permanentemente seu corpo técnico, em especial os profissionais de alto desempenho que estão presentes e atuantes em todo território nacional.

Havia a necessidade de um modelo na construção de um espaço informacional multimodal para capacitação de profissionais de alto desempenho. O modelo DAIM:CSCL, que até o momento é um modelo teórico, foi o escolhido, entre outros motivos, devido à sua divisão por fases e sua característica cíclica de implementação. Essa foi sua a primeira aplicação prática. Este modelo foi desenvolvido como tese de doutorado na Faculdade de Ciência da Informação - FCI, da Universidade de Brasília - UNB, e apresentado no X Encontro de Docentes de Escolas de Biblioteconomia e Ciência da Informação do MERCOSUL - EBCIM 2016 (STEINMETZ et al., 2016). 
As fases do modelo DAIM:CSCL, foram aplicadas da seguinte forma:

- Iniciação: a demanda surgiu do curso de Curso de Pós-Graduação (Especialização) a Distância em Gestão de Negócios, elaborado para atender à formação de profissionais de alto desempenho, já graduados, na disciplina Gestão de Projetos;

- Planejamento: foi decidida a utilização de um Ambiente Virtual de Aprendizagem AVA, pois as turmas estão localizadas nos estados da federação e tem-se a necessidade de disponibilizar o material de forma equânime e simultânea para todo o corpo discente. Foi escolhido o ambiente Moodle como AVA, por se tratar de um software livre, de fácil instalação e configuração, que contempla as camadas de apresentação e persistência em um mesmo ambiente. Não foi necessária a aquisição de nenhum outro hardware ou software para implementação da solução.

- Implementação: nesta fase foi construído o espaço informacional multimodal, sobre a plataforma Moodle, como apresentado no exemplo das figuras 2 e 3. Para esta construção foram utilizadas as funcionalidades da plataforma de software escolhida, o Moodle;

- Teste Piloto: foram elaborados os objetos multimodais de aprendizagem, como apresentações e textos. Os demais objetos multimodais, vídeos e áudios, foram baixados de sítios específicos sobre o tema (gerenciamento de projetos), definidos na fase de planejamento, e inseridos no espaço informacional multimodal suportado pelo AVA. Antes do início efetivo da disciplina, o ambiente foi testado por parte do corpo docente;

- Implantação e Manutenção: o ambiente foi disponibilizado para todos os alunos, distribuídos nos estados da federação, que puderam fazer uso da plataforma AVA e dar sugestões de melhoria no ambiente.

- Análise dos Resultados: neste artigo estão sendo apresentadas os primeiros resultados da aplicação do modelo. Após algumas análises iniciais será possível implementar futuras mudanças, de forma a melhorar a qualidade, eficiência e eficácia, aprimorando a execução e corrigindo eventuais falhas.

Como primeira implementação do modelo DAIM:CSCL foi construído o espaço informacional multimodal - Gestão de Projetos, elaborado no AVA (Moodle) para capacitação de profissionais de alto desempenho, do curso de pós-graduação em Gestão de Negócios em uma organização estatal de grande porte. A disciplina Gestão de Projetos foi ministrada à distância para 129 alunos localizados nos estados do país. O corpo de discentes da disciplina foi composto de profissionais de alto desempenho, uma vez que conforme processo seletivo interno da 
organização, todos têm um perfil de atuação destacada e buscam a obtenção de resultados para a organização em que atuam, seus clientes e outros profissionais com os quais trabalham.

O modelo DAIM:CSCL foi adotado para que possa ser estudada sua eficácia como um modelo a ser evoluído e empregado na capacitação de profissionais de alto desempenho. As figuras 3 e 4 apresentam uma visão geral do Espaço Informacional Multimodal - Gestão de Projetos. A figura 3 apresenta a página principal da disciplina, em que são apresentados os objetos multimodais que compõe todo o material didático disponibilizado para os discentes.

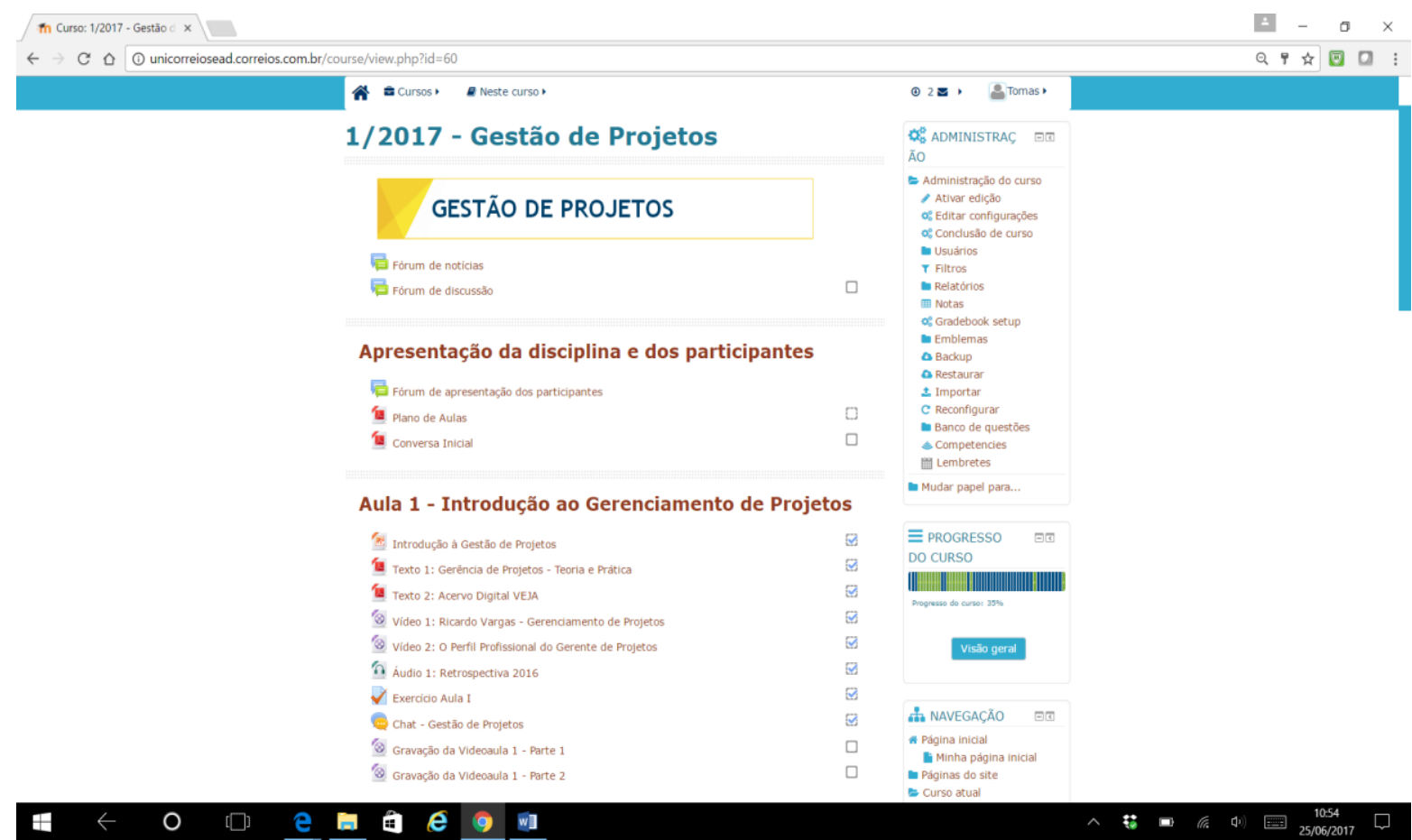

Figura 3: Espaço Informacional Multimodal - Gestão de Projetos: Página principal. Fonte: ambiente de pesquisa desenvolvido pelos autores

A página principal do ambiente contém os vários objetos informacionais multimodais (apresentações, textos, áudios, vídeos didáticos, videoaulas, etc.) que são escolhidos e direcionam para uma próxima página (figura 3) que disponibiliza o objeto multimodal específicos selecionado. 


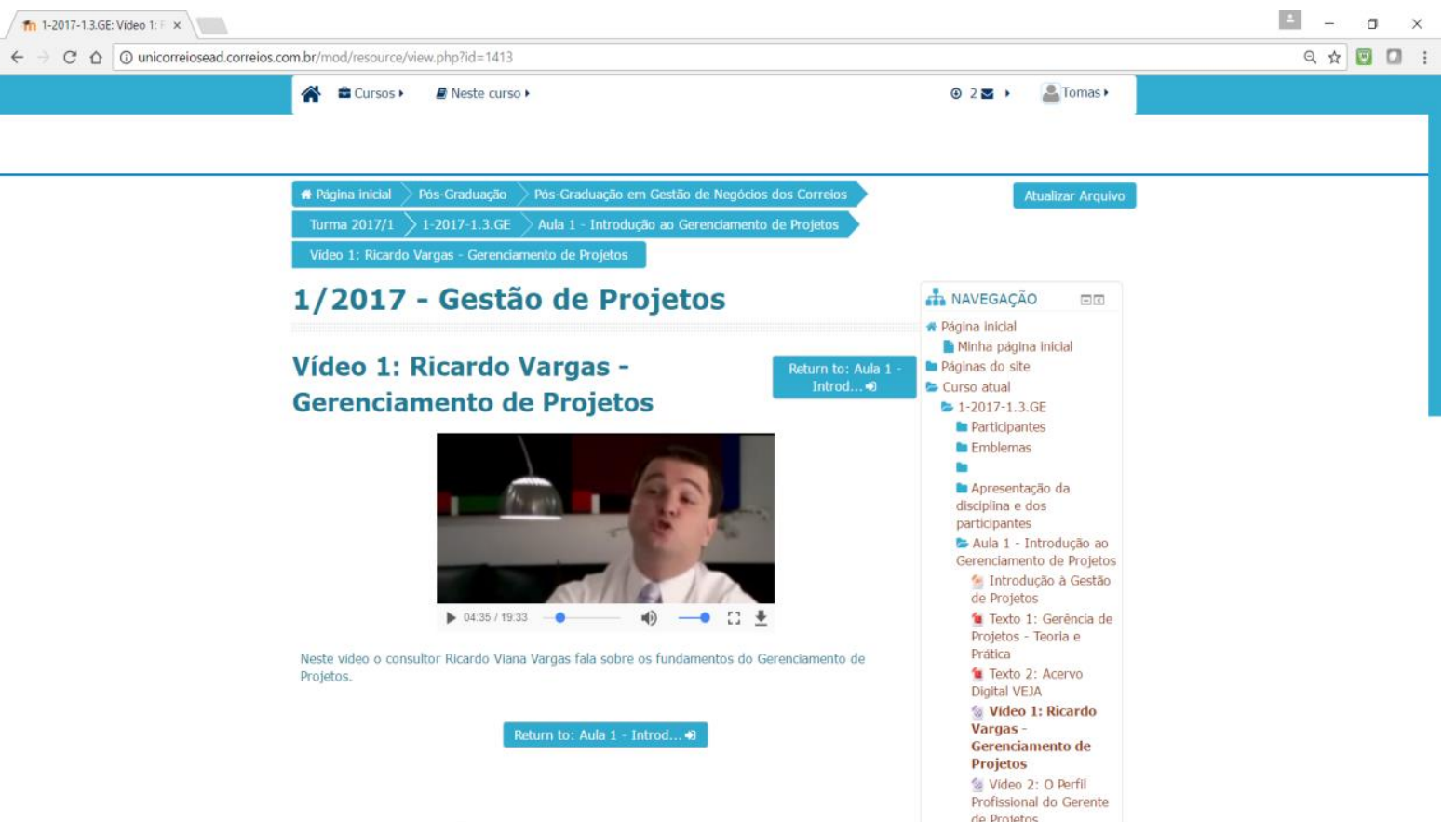

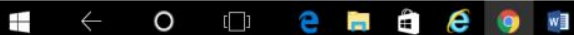

Figura 4: Espaço Informacional Multimodal - Gestão de Projetos: Página específica. Fonte: ambiente de pesquisa desenvolvido pelos autores

\section{Resultados}

A pesquisa foi realizada de 01 a 17/07/2017, período que foi disponibilizado o questionário para os discentes da disciplina Gestão de Projetos do curso de pós-graduação em Gestão de Negócios. A disciplina teve a participação de 129 alunos e a pesquisa foi respondida por 119, representando um índice de $92 \%$ de respostas válidas. A tabela 1 apresenta a escala utilizada na avaliação de reação dos alunos participantes.

Tabela 1: Escala de Avaliação.

\begin{tabular}{l|c}
\hline \hline Nota & Valor \\
\hline \hline Ruim & $1-2-3$ \\
\hline Regular & $4-5-6$ \\
\hline Bom & $7-8$ \\
\hline Muito Bom & 9 \\
\hline Ótimo & 10 \\
\hline \hline
\end{tabular}

Fonte: ambiente de pesquisa desenvolvido pelos autores

Foram elaboradas dez perguntas sobre o programa apresentado, sendo cinco perguntas específicas sobre objetos multimodais disponibilizados para o aprendizado dos discentes. Nestas questões foi explicado que as "necessidades de informação", são a formação do hábito de 
indivíduos na busca da informação, assim como os usos da documentação e da informação para solução de um problema. No caso da disciplina Gestão de Projetos, as necessidades de informações dos alunos são os itens necessários para: compreender a temática apresentada, solucionar os exercícios disponibilizados, responder às questões da prova e elaborar o trabalho final da disciplina:

- Pergunta 1: O arquivo apresentação (power point) disponibilizado atendeu às minhas necessidades de informação nesta disciplina.

\section{Pergunta 1}

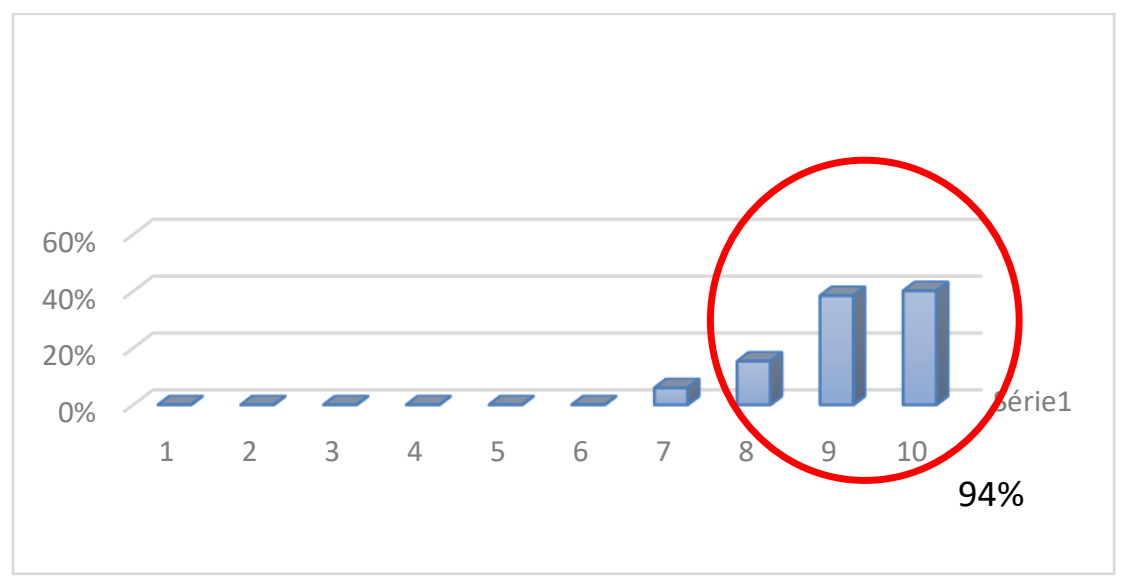

\section{Gráfico 1: Pergunta 1}

Fonte: pesquisa desenvolvida pelos autores

- Pergunta 2: Os arquivos de textos (.doc e .pdf) disponibilizados atenderam às minhas necessidades de informação nesta disciplina.

\section{Pergunta 2}

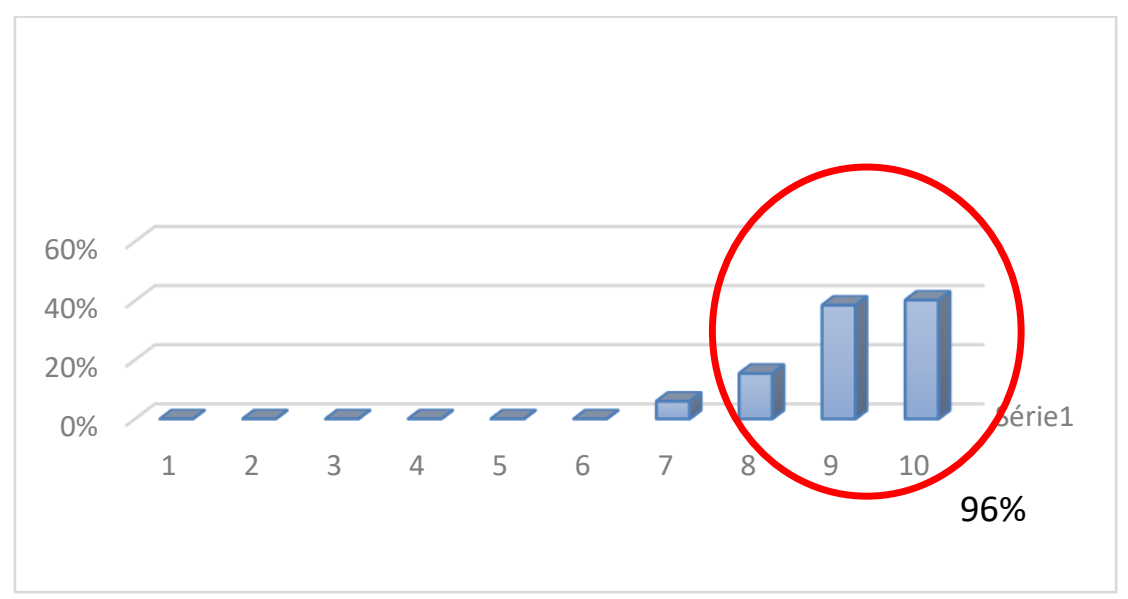

Gráfico 2: Pergunta 2

Fonte: pesquisa desenvolvida pelos autores 
As respostas às perguntas 1 e 2 apresentaram, respectivamente, um resultado de $94 \% \mathrm{e}$ 96\% de concordância para as notas de 8 a 10, conceitos de bom a ótimo para as perguntas

As perguntas 3,4 e 5 somaram $95 \%$ de aprovação na escala de notas 8,9 ou 10 :

- Pergunta 3: Os arquivos de vídeos (.mp4) disponibilizados atenderam às minhas necessidades de informação nesta disciplina.

- Pergunta 4: Os arquivos de áudio (.mp3) disponibilizados atenderam às minhas necessidades de informação nesta disciplina.

- Pergunta 5: As videoaulas disponibilizadas atenderam às minhas necessidades de informação nesta disciplina.

Perguntas 3,4 e 5

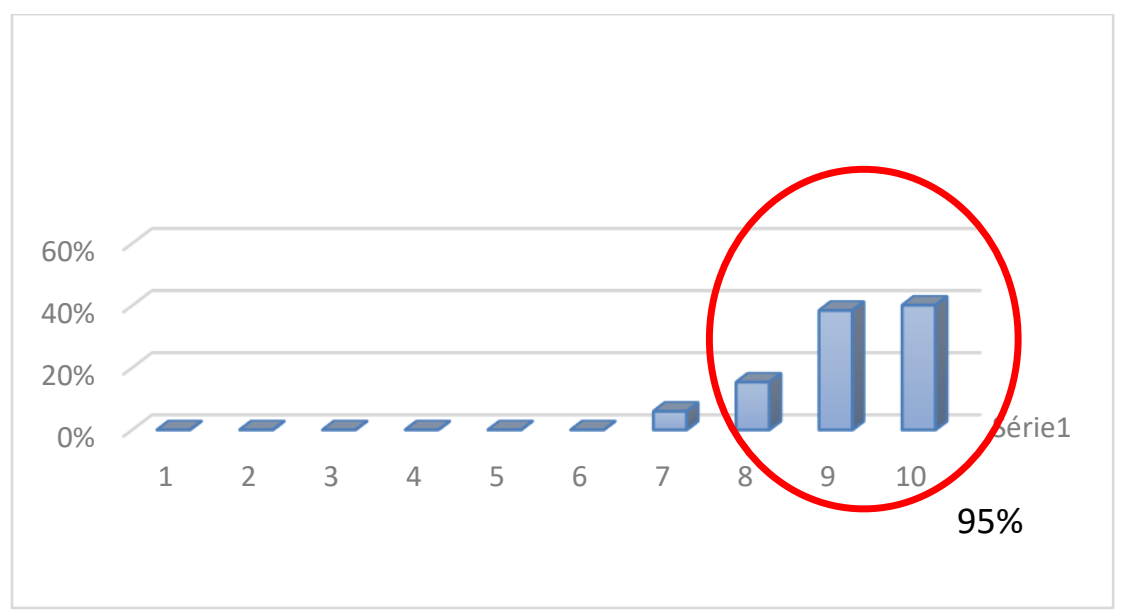

Gráfico 3: Perguntas 3,4 e 5

Fonte: pesquisa desenvolvida pelos autores

Foi também elaborada a seguinte pergunta aberta sobre a disciplina: "Quais os pontos de destaque ou oportunidades de melhoria? ". A seguir estão listadas algumas respostas transcritas do AVA da disciplina Gestão de Projetos:

1. "Utilizar a didática desta matéria para as demais.";

2. “...o uso de tecnologias auxiliam a fixar conceitos. Resolvi questão da prova lembrando das situações da aula.";

3. "Esta matéria foi muito bem elaborada pelos professores, podendo ser adotada como modelo para outras matérias. "; 
4. "Um dos pontos destaque foi a gravação e disponibilização das aulas, para que os alunos possam revisar algo que tenha passado despercebido. Sugiro que essa melhoria possa ser implantada em outras disciplinas.";

5. "Acredito que os vídeos das aulas terem sido disponibilizados no AVA foi um ponto forte, pois serve de auxilio nos estudos. ";

6. "Ponto de Destaque: Aulas dinâmicas, o conteúdo de apoio, principalmente os vídeos e áudios foram muito bons e acrescentaram bastante no conteúdo final “;

7. “Os vídeos e áudios disponibilizados ajudaram muito no entendimento do conteúdo, bem esclarecedores e concisos ";

8. “Os pontos de destaque foram os vídeos e os áudios ";

9. “Adorei os vídeos e áudios que além de enriquecer o meu conhecimento tornou meu aprendizado mais dinâmico “;

10. "Ponto de Destaque foi a gravação das aulas, pois o aluno tinha a oportunidade de assistir a vídeo conferência e ainda tinha a oportunidade de assistir novamente a vídeo-aula “.

As respostas à última pergunta da pesquisa destacam que as utilizações dos objetos de aprendizagem multimodais tiveram boa aceitação do corpo discente, e foram entendidos como um diferencial relevante no aprendizado da disciplina ministrada.

\section{Considerações finais}

A capacitação de profissionais é um desafio constante, especialmente dos quais são exigidas atuações destacadas e de alto desempenho. A proposta de construção de um modelo de espaço informacional para apoiar substancialmente a formação desses profissionais é o principal objeto dessa pesquisa.

A multimodalidade aplicada na educação desses profissionais, no ponto de vista dos autores, pode auxiliar significativamente no alcance das suas necessidades de informação para desempenhar suas atividades técnicas e gerenciais. A presente pesquisa utilizou conceitos de arquitetura da informação, espaços informacionais e multimodalidade para avaliar a efetividade de um modelo de ensino e aprendizagem, concebido para o ensino aplicado à distância, como principal instrumento de capacitação de profissionais de alto desempenho.

Os resultados demonstrados no item resultados, dentro dos limites dessa pesquisa, levam a conclusão que o Espaço Informacional Multimodal elaborado pode ser uma resposta ao 
principal questionamento de como uma Arquitetura da Informação associada a Multimodalidade pode auxiliar na capacitação de profissionais de alto desempenho, uma vez que o espaço elaborado atendeu às necessidades de informação de grande parte dos profissionais de alto desempenho, discentes da disciplina avaliada. A aplicação do modelo DAIM:CSCL na construção do espaço informacional multimodal suportou as demandas do discentes e apresentou boa avaliação geral.

As limitações da pesquisa são: a aplicação dos conceitos apresentados em apenas uma disciplina, não sendo possível estabelecer comparações com outras disciplinas com aplicações dos mesmos conceitos, e também não foram pesquisadas outras disciplinas que não fizeram uso dos mesmos recursos. Tais limitações foram estabelecidas pelos pesquisadores com o intuito de isolar as avaliações em apenas uma primeira aplicação dos conceitos de Arquitetura da Informação associada a Multimodalidade.

Como pesquisas futuras, consideramos que, por ser esta a primeira aplicação dos conceitos apresentados na capacitação de profissionais de alto desempenho, as sugestões para próximos trabalhos são muitas. Primeiramente repetir o experimento com outro corpo discente, igualmente com perfil de alto desempenho, para verificar se as necessidades de informação foram atendidas de forma semelhante. Devem também ser estabelecidas comparações com outras disciplinas que não fizeram uso dos mesmos recursos, para verificar se os resultados são semelhantes ou não. Finalmente o objetivo maior desta pesquisa é a proposição de um modelo que possa auxiliar significativamente na capacitação das competências requeridas aos de profissionais de alto desempenho. 


\section{Referências}

BARROS, D. L. P. Teoria semiótica do texto. São Paulo: Ática, 2005.

BUCKLAND, M. K. Information as thing. Journal of the American Society for Information Science (1986-1998), v. 42, n. 5, p. 351-360, 1991.

CAMARGO, L. S. A. Metodologia de desenvolvimento de ambientes informacionais digitais a partir dos princípios da arquitetura da informação. 2010. $289 \mathrm{f}$. Tese (Doutorado) - Universidade Estadual Paulista, Faculdade de Filosofia e Ciências, 2010.

CAPURRO, R. Epistemología y Ciencia de la Informacíon. Enl@ ce: revista Venezolana de Informacíon, Tecnología y Conocimiento, año 4, n.1, Enero-Abril, p. 11-29, 2007.

DENZIN, N. K.; LINCOLN, Y. S. Planejamento da pesquisa qualitativa: teorias e abordagens. Porto Alegre: Artmed, 2006. p. 367-388.

DURAND, T. Forms of incompetence. In: INTERNATIONAL CONFERENCE ON COMPETENCE-BASED MANAGEMENT, 4., 1998. Proceedings... Oslo, 1998.

DIONISIO, A. P. Gêneros textuais e multimodalidade. In: KARWOSKI, A. M.; GAYDECZKA, B.; BRITO, K. S. (Org.). Gêneros textuais: reflexões e ensino. São Paulo: Parábola, 2011.

Multimodalidade discursiva na atividade oral e escrita (atividades). In:

MARCUSCHI, L. A.; DIONISIO, A. P. (Org.). Fala e escrita. Belo Horizonte: Autêntica, 2005.

GONZÁLEZ DE GÓMEZ, M. N. O objeto de estudo da Ciência da Informação: paradoxos e desafios. Ciência da Informação, Rio de Janeiro, v. 19, n. 2, p. 117-122, jul./dez. 1990.

HJORLAND, B. Epistemology and the socio-cognitive perspective in information science. Journal of the American Society for Information Science and Technology, v. 53, n. 4, p. 257-270, 2002.

KRESS, G.; VAN LEEUWEN, T. Multimodal discourse: the models and media of contemporary communication. London: Edward Arnold, 2001.

LYRA, M. R. A contribuição da arquitetura da informação para o gerenciamento de serviços de TI. 2012. Tese (Doutorado) - Programa de Pós-Graduação em Ciência da Informação, Universidade de Brasília, 2012.

LOSADA, M. F. The complex dynamics of high performance teams. Journal Mathematical and Computer Modelling, v. 30, 179-192, 1999.

LUNA, T. S. A pluralidade de vozes em aulas e artigos científicos. Revista Ao Pé da Letra, Recife, v. 4, 2002. 
MACEDO, F. L. O. Arquitetura da Informação: aspectos epistemológicos, científicos e práticos. 186f. 2005. Dissertação (Mestrado em ciência da informação). Universidade de Brasília, Brasília, 2005.

MAZURKIEWICZ, L.G. O papel da empresa no desenvolvimento da carreira do profissional considerado de alto desempenho. In: SEMEAD - SEMINÁRIOS EM ADMINISTRAÇÃO, 13., 2010. Gestão de pessoas. São Paulo: FEA/USP, 2010.

OLIVEIRA, C. B. Uma proposta de arquitetura da informação para o processo de inovação em centros de pesquisa. 2012. 353 f. Tese (Doutorado) - Programa de PósGraduação em Ciência da Informação. Universidade de Brasília, Brasília, 2012.

PINHEIRO, L.V. Fronteiras e horizontes da pesquisa em ciência da informação no Brasil. Brasília: IBICT, 2013.

PRAHALAD, C. K.; HAMEL, G. The core competence of the corporation. Harvard Business Review, v. 68, n. 3, 1990.

SARACEVIC, T. A natureza interdisciplinar da ciência da informação. Ciência da Informação, Brasília, v. 24, n. 1, 1995.

RICHARDSON, R. J. Pesquisa social: métodos e técnicas. São Paulo: Atlas, 2008.

SEI (Software Engineering Institute). People CMM Model - P-CMM. Disponível em: <https://cmmiinstitute.com/products/cmmi/pm>. Acesso em: 16 jul. 2017.

VARGAS, R. V. Construindo times altamente eficazes: como transformar equipes virtuais em redes profissionais eficazes e coesas. Rio de Janeiro: Brasport, 2014.

XAVIER, A. C. Como se faz um texto: a construção da dissertação argumentativa. Catanduva: Rêspel, 2006.

STEINMETZ, E.; DUQUE, C. G.; ORLANDI, T. R. C. O. Contribuições da arquitetura da informação para ambientes de colaborativos de ensino-aprendizagem. In: Encontro de Docentes de Escolas de Biblioteconomia e Ciência da Informação do Mercosul - EBCIM 2016, Anais... Belo Horizonte: UFMG, 2016. Disponível em: <http://mercosur2016.eci.ufmg.br/wp-content/uploads/2016/10/AnaisMercosur5.pdf $>$. Acesso em: 20 jul. 2017. 